\title{
Cardiovascular disease and cumulative incidence of cognitive impairment in the Health and Retirement Study
}

\author{
Allyson L. Covello ${ }^{1 *}$, Leora I. Horwitz² ${ }^{2}$ Shreya Singhal ${ }^{3}$, Caroline S. Blaum ${ }^{4}$, Yi Li ${ }^{5}$ and John A. Dodson²,6
}

\begin{abstract}
Background: We sought to examine whether people with a diagnosis of cardiovascular disease (CVD) experienced a greater incidence of subsequent cognitive impairment (CI) compared to people without CVD, as suggested by prior studies, using a large longitudinal cohort.

Methods: We employed Health and Retirement Study (HRS) data collected biennially from 1998 to 2014 in 1305 U.S. adults age $\geq 65$ newly diagnosed with CVD vs. 2610 age- and gender-matched controls. Diagnosis of CVD was adjudicated with an established HRS methodology and included self-reported coronary heart disease, angina, heart failure, myocardial infarction, or other heart conditions. Cl was defined as a score $\leq 11$ on the 27-point modified Telephone Interview for Cognitive Status. We examined incidence of $\mathrm{Cl}$ over an 8-year period using a cumulative incidence function accounting for the competing risk of death.

Results: Mean age at study entry was 73 years, 55\% were female, and 13\% were non-white. Cognitive impairment developed in 1029 participants over 8 years. The probability of death over the study period was greater in the CVD group (19.8\% vs. 13.8\%, absolute difference 6.0, 95\% confidence interval 2.2 to $9.7 \%$ ). The cumulative incidence analysis, which adjusted for the competing risk of death, showed no significant difference in likelihood of cognitive impairment between the CVD and control groups (29.7\% vs. 30.6\%, absolute difference $-0.9,95 \%$ confidence interval -5.6 to 3.7\%). This finding did not change after adjusting for relevant demographic and clinical characteristics using a proportional subdistribution hazard regression model.
\end{abstract}

Conclusions: Overall, we found no increased risk of subsequent $\mathrm{Cl}$ among participants with CVD (compared with no CVD), despite previous studies indicating that incident CVD accelerates cognitive decline.

Keywords: Cardiovascular disease, Cognition, Geriatric cardiology

\section{Background}

Cardiovascular disease (CVD) is a common chronic condition that disproportionately affects older adults [1]. Prior studies have shown a connection between CVD and cognitive impairment [2-8], with multiple potential mechanisms at play, including atherosclerotic-related cerebrovascular disease (causing cerebral hypoxia, brain

\footnotetext{
*Correspondence: allyson.covello@nyulangone.org

${ }^{1}$ New York University Grossman School of Medicine, 550 First Avenue, New York, NY, USA

Full list of author information is available at the end of the article
}

infarctions, and damage to the blood-brain barrier) [9-11]; oxidative stress and inflammatory immune responses $[12,13]$; and thromboembolism from concomitant atrial fibrillation [14, 15]. More recently, several longitudinal studies have indicated that incident CVD is associated with an acceleration in the decline in memory and processing speed that occurs normally with age [16-18]. This research suggests that onset of CVD may serve as a cognitive 'inflection point,' whereby incident CVD leads to a more rapid deterioration in patients'

(c) The Author(s). 2021 Open Access This article is licensed under a Creative Commons Attribution 4.0 International License, which permits use, sharing, adaptation, distribution and reproduction in any medium or format, as long as you give appropriate credit to the original author(s) and the source, provide a link to the Creative Commons licence, and indicate if changes were made. The images or other third party material in this article are included in the article's Creative Commons licence, unless indicated otherwise in a credit line to the material. If material is not included in the article's Creative Commons licence and your intended use is not permitted by statutory regulation or exceeds the permitted use, you will need to obtain permission directly from the copyright holder. To view a copy of this licence, visit http://creativecommons.org/licenses/by/4.0/ The Creative Commons Public Domain Dedication waiver (http://creativecommons.org/publicdomain/zero/1.0/) applies to the data made available in this article, unless otherwise stated in a credit line to the data. 
cognition. However, non-significant associations have also been reported [19-21], and inconsistencies in definition of CVD, cognitive assessment tools, and follow-up duration complicate reaching generalized conclusions across studies.

Further understanding of the relationship between CVD and cognitive impairment is important in informing targeted screening and prevention efforts for cognitive impairment in vulnerable subpopulations of older adults. As such, we designed our present study to examine whether cognitively normal older adults with a diagnosis of CVD experienced a greater incidence of subsequent cognitive impairment, measured using an easily administered global assessment of cognitive function, compared to people without a diagnosis of CVD. We used data from the Health and Retirement Study (HRS), a large, well-characterized cohort study with a long duration of follow-up, an important feature given that many of the proposed biologic mechanisms connecting CVD and cognitive impairment are slow-acting processes. In order to limit the potential for bias from to high rates of attrition due to death, we used a cumulative incidence function to analyze the likelihood of cognitive impairment while accounting for the competing risk of death.

\section{Methods}

\section{Data}

We used data from the 1998-2014 waves of HRS. HRS is a longitudinal panel study that surveys $20,000+$ Americans, nearly all of whom are age 50 or older, every 2 years [22]. The HRS survey population is largely representative of the U.S., with slight oversampling of Black, Hispanic, and Floridian households. Questions address physical health and mental health, as well as demographic characteristics like age, marital status, and level of education.

\section{Definition of CVD group}

To define the diagnosis of CVD, we used participant self-report based on the question, "Has a doctor told you that you have had a heart attack, coronary heart disease, angina, congestive heart failure, or other heart problems?" This definition of CVD encompassed the non-stroke cardiovascular conditions, such as heart failure, myocardial infarction, atrial fibrillation, and coronary artery disease, shown in prior research to impact cognitive function $[4,6,7,16,18]$. The year of CVD diagnosis was defined as the first survey wave that a participant responded 'yes' to this question, after having responded 'no' in previous waves. To adjust for the potential inconsistencies in self-reported data from longitudinal surveys, we employed an adjudication method previously developed for refining participants' responses to HRS questions about chronic disease, including CVD [23]. We then excluded anyone from the control group who was missing important demographic or cognition data, who had inconsistencies in their age reporting survey to survey, or who had cognitive impairment at or before baseline. We also excluded participants who were less than 65 at baseline, since the tool we used to assess cognitive status was not validated in a population under 65 , as well as participants who were greater than 85 years old to enable appropriate generation of a matched control group per below.

\section{Definition of the control group}

To develop a comparator control group (without CVD), we needed to define a 'baseline' year for control participants that could be matched to the 'CVD diagnosis' year for our CVD group. Because our participants diagnosed with CVD during the study period had different years of diagnosis, we used an age-matching control-generation process that pulled control participants that were the same age as CVD participants in their year of diagnosis and defined that year as 'baseline' for the control participants. We generated the control at a 2:1 ratio and also matched on gender to compensate for the fact that the overall HRS population skewed more female than the CVD population. We used the nearest neighbor matching methodology to generate the age-and gender-matched control, instead of an exact matching methodology, to account for the fact that matching to an exact year was not possible in the $80+$ age range. As we did with our CVD group, we excluded anyone from the control group who was missing important demographic or cognition data, who had inconsistencies in their age reporting survey to survey, who was less than 65 or greater than 85 years old at baseline, or who had cognitive impairment at or before baseline.

When we completed the match, there was no significant difference in age and gender at baseline between our CVD and control groups, confirming that our matching process was successful. The complete participant selection process, including the adjudication method for CVD, the method for generating a matched control group, and the individuals removed from our final study sample, is further described in the Supplementary Methods and in Supplementary Figure S1.

\section{Assessment of cognitive status}

The presence of cognitive impairment was determined using a modified version of the Telephone Interview for Cognitive Status (TICS-m). The TICS- $m$ is a validated cognitive screening tool based on the Mini-Mental State Examination that has been used in prior research on the connection between CVD and cognitive impairment [5]. Unlike the Mini-Mental State Examination, the TICS-m does not need to be administered in person. The TICS-m used in HRS includes questions about immediate and delayed word recall to assess memory, serial seven subtraction to assess working memory, and counting backwards 
to assess information processing speed. It has high sensitivity and specificity for cognitive impairment in older adults $[24,25]$ and is measured on a 27-point scale, where a score of 11 or lower indicates cognitive impairment [26]. Onset of cognitive impairment was marked in the first survey wave that a participant had a TICS-m score of 11 or lower.

Some survey respondents in a given wave could not participate in the interview due to physical or mental limitations and instead used a proxy respondent. For those respondents, HRS offered an alternative measure of cognitive status using information from the proxy and the interviewer as to the interviewee's cognitive status [27]. To avoid preferentially excluding cognitively impaired participants, who are more likely to have missing TICS values, we used the Langa-Weir cognition data from the HRS Survey Research Center, which has imputed cognition values for missing responses and is the standard dataset used for HRS cognitive assessments [28]. Additional information on cognitive assessment tools is provided in the Supplementary Methods.

\section{Covariates}

Covariates were defined at baseline and included race and ethnicity, years of education, marital status, body mass index, current smoker, riskiness of drinking behavior, presence of depressive symptoms, and comorbid chronic conditions (hypertension, cancer, chronic lung disease, diabetes). These covariates were determined based on prior literature examining the association between CVD and cognitive impairment [16-18] and clinical judgement of study investigators.

\section{Statistical analysis}

To examine the incidence of cognitive impairment in the CVD vs. control groups, we used a cumulative incidence function, which is a type of time-to-event analysis that looks at the probability of occurrence of an event of interest over a defined period of time [29]. Because our population was compromised of older adults, many of whom had multiple chronic health conditions, we incorporated into our analysis the competing risk of death, which takes into account the fact that death precludes our event of interest from ever occurring. This prevents the overestimation of probability of event occurrence that can happen when death is not accounted for [30]. We also calculated a proportional subdistribution hazard regression model to adjust for covariates while simultaneously accounting for competing risk [29].

Additionally, we performed 2 sensitivity analyses. In the first sensitivity analysis, we redefined our exclusion criteria to be less stringent and included in the CVD group participants previously excluded due to self-report discrepancies. We then generated a new matched control and repeated the cumulative incidence function with the new CVD and control groups. In the second, we redefined the threshold for cognitive impairment using TICS-m cutoffs of 10 and 12 instead of 11 and reran the cumulative incidence function with the new thresholds.

All data cleaning and analyses were conducted using RStudio (RStudio, Inc. 2020) [31]. The cumulative incidence function was generated using the cmprsk package [32]. All analyses used publicly available, non-restricted data collected through the Health and Retirement Survey and did not require IRB/human subjects review.

\section{Results}

\section{Baseline characteristics and sample size}

The final sample consisted of 1305 participants with incident CVD and 2610 age- and gender-matched controls. Mean age at study entry was 73 years, and $55 \%$ were female. $87 \%$ of participants were non-Hispanic white, $5 \%$ were Hispanic, and $7 \%$ were non-Hispanic Black (Table 1). Mean length of follow-up was 5.01 years (standard deviation $=1.49$ ) in the incident CVD group and 5.33 years (standard deviation $=1.35$ ) in the control group. The mean TICS-m scores at baseline were not significantly different between the incident CVD and control groups (16.6, 95\% confidence interval 16.5 to 16.8 vs. $16.8,95 \%$ confidence interval 16.7 to 16.9 ), and trended downwards at the same rate during the 8-year study period (Fig. 1). The TICS-m scores for CVD and control groups had similar distributions at baseline and at years 2, 4, 6 and 8 (Supplementary Figure S2).

The number of participants at risk of cognitive impairment in the CVD group was 1305 (100\%), 1062 (81\%), 769 (59\%), and $487(37 \%)$ at $2,4,6$, and 8 years. The number of participants at risk of cognitive impairment in the control group was 2610 (100\%), 2182 (84\%), 1570 $(60 \%)$, and $1054(40 \%)$ at $2,4,6$, and 8 years.

\section{Cumulative incidence of cognitive impairment and death}

We examined the cumulative incidence of cognitive impairment in the CVD and control groups over the 8-year study period, accounting for the competing risk of death. During the study period, cognitive impairment developed in 339 (26.0\%) of participants in the CVD group and $690(26.4 \%)$ of participants in the control group. Death before cognitive impairment occurred in 232 (17.8\%) of participants in the CVD group and 317 (12.1\%) of participants in the control group. Per the cumulative incidence analysis, the probability of death over 8 years was greater in the CVD group $(19.8 \%$ vs. $13.8 \%$, absolute difference $6.0,95 \%$ confidence interval 2.2 to $9.7 \%$ ). The probability of cognitive impairment accounting for the competing risk of death over 8 years was not significantly different between the CVD and 
Table 1 Baseline Sample Size and Cohort Characteristics

\begin{tabular}{|c|c|c|}
\hline Variable & $\begin{array}{l}\text { Incident CVD Group } \\
n=1305\end{array}$ & $\begin{array}{l}\text { Matched Control Group } \\
n=2610\end{array}$ \\
\hline Age (yrs) & $73.3(5.63)$ & $73.3(5.64)$ \\
\hline Women & $702(53.8 \%)$ & $1455(55.7 \%)$ \\
\hline \multicolumn{3}{|l|}{ Race \& Ethnicity } \\
\hline Non-Hispanic White & 1170 (89.7\%) & $2218(85.0 \%)$ \\
\hline Non-Hispanic Black & $69(5.3 \%)$ & $193(7.4 \%)$ \\
\hline Hispanic & $50(3.8 \%)$ & $154(5.9 \%)$ \\
\hline Other & $16(1.2 \%)$ & $45(1.7 \%)$ \\
\hline \multicolumn{3}{|l|}{ Education level } \\
\hline Less than high school & $179(13.7 \%)$ & $381(14.6 \%)$ \\
\hline High school & $478(36.6 \%)$ & $952(36.5 \%)$ \\
\hline More than high school & $648(49.7 \%)$ & 1277 (48.9\%) \\
\hline $\mathrm{BMI}(\mathrm{kg} / \mathrm{m} \wedge 2)$ & $27.4(5.54)$ & $26.7(4.75)$ \\
\hline Married & $819(62.8 \%)$ & $1684(64.5 \%)$ \\
\hline Depressive symptoms & $163(12.5 \%)$ & 207 (7.9\%) \\
\hline Smoking currently & $102(7.8 \%)$ & $237(9.1 \%)$ \\
\hline \multicolumn{3}{|c|}{ Drinking behavior (past 3 mos) } \\
\hline Not drinking & $891(68.3 \%)$ & $1574(60.3 \%)$ \\
\hline Low-risk drinker & $402(30.8 \%)$ & 1015 (38.9\%) \\
\hline High-risk drinker & $12(0.9 \%)$ & $21(0.8 \%)$ \\
\hline Hypertension & $927(71.0 \%)$ & $1431(54.8 \%)$ \\
\hline Diabetes & $324(24.8 \%)$ & $400(15.3 \%)$ \\
\hline Chronic lung disease & $182(13.9 \%)$ & 205 (7.9\%) \\
\hline Cancer & $278(21.3 \%)$ & $515(19.7 \%)$ \\
\hline TICS-m score & $16.6(2.87)$ & $16.8(2.91)$ \\
\hline
\end{tabular}

Values are mean + - standard deviation or $\mathrm{n}(\%)$. Race and ethnicity is defined based on participants' primary response, and 'other' for race and ethnicity is defined as American Indian, Alaska Native, Asian, Native Hawaiian, Pacific Islander, or something else. High-risk drinking is defined as more than 7 drinks per week or 3 drinks per day for women, or more than 14 drinks per week or 4 drinks per day for men on average over the past 3 months Abbreviations: CVD cardiovascular disease, BMI body mass index, TICS- $m$ modified Telephone Interview for Cognitive Status

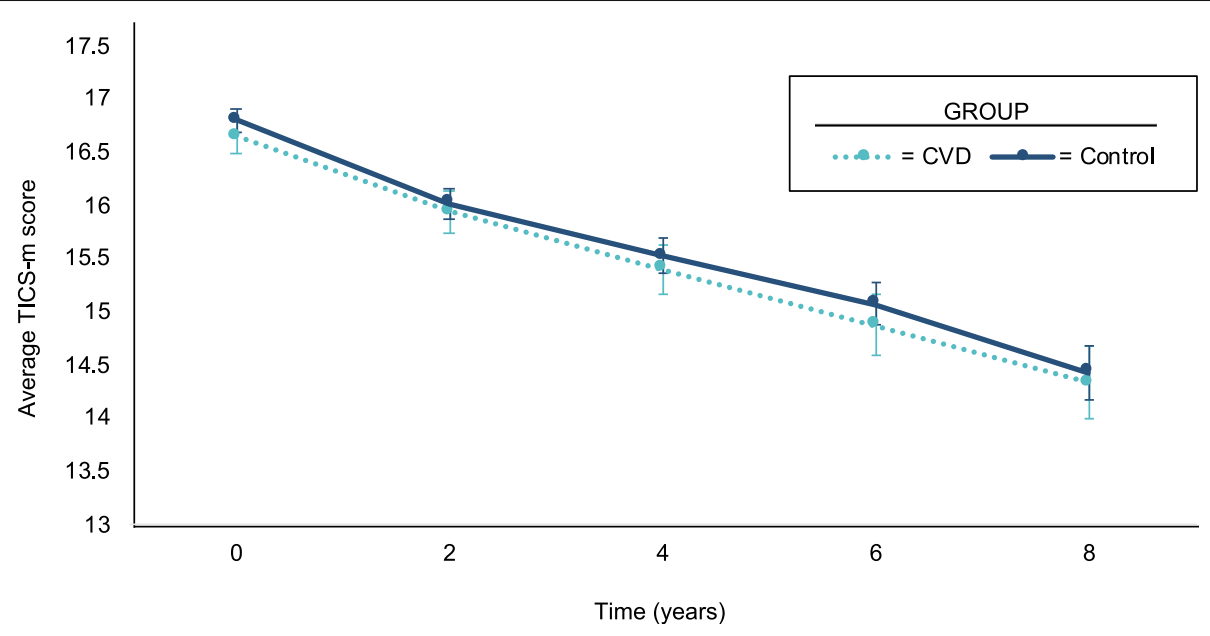

Fig. 1 Trend in Mean Modified Telephone Interview for Cognitive Status Score Over Time. Shown are the mean TICS-m scores in the CVD group (dashed light blue line) vs. control group (solid dark blue line) over the 8-year study period. Bars represent 95\% confidence intervals 
control groups $(29.7 \%$ vs. $30.6 \%$, absolute difference $0.9,95 \%$ confidence interval -5.6 to $3.7 \%$ ) (Fig. 2).

\section{Subdistribution hazard regression model of incidence of cognitive impairment and death}

Given the potential effect of covariates on the cumulative incidence of cognitive impairment, we created a proportional subdistribution hazard regression model, which assesses the effect of multiple variables on incidence of a particular event in a competing risk analysis. The subdistribution hazard ratios and their associated 95\% confidence intervals describe whether a particular variable has a significant effect on the outcome of interest, such as cognitive impairment, and the direction of this relationship (i.e. increases or decreases outcome incidence), though the magnitude of the ratio cannot be inferred as representing the relative increase in incidence of the outcome of interest [33].

Based on our proportional subdistribution hazard regression model, when incorporating covariates of race and ethnicity, education, body mass index, marital status, depressive symptoms, current smoking, level of alcohol intake, and chronic conditions (hypertension, diabetes, chronic lung disease, and cancer) into the analysis, incident CVD remained not significant in prognosticating incidence of cognitive impairment (subdistribution hazard ratio $=.96,95 \%$ confidence interval .85 to 1.09 ). The subdistribution hazard ratios and 95\% confidence intervals for each variable in the model are further outlined in Table 2 .

\section{Sensitivity analyses}

One hundred eighty-nine participants who met other inclusion criteria (not cognitively impaired before CVD diagnosis, not missing key cognition or covariate data, and between 65 and 85 at time of CVD onset) were excluded from the main analysis due to unresolved discrepancies in CVD self-report. These 189 participants with self-report discrepancies were more likely to develop cognitive impairment during the study period (41.3\% vs. $26.0 \%)$ and less likely to experience death before cognitive impairment (7.9\% vs. 17.8\%) (Supplementary Table S1). We then re-examined the cumulative incidence of cognitive impairment in the CVD and control groups accounting for the competing risk of death, including these 189 participants. This new sample consisted of 1494 participants with incident CVD and 2988 age- and gender-matched controls. Per the cumulative incidence analysis, the probability of death over 8 years was still greater in the CVD group than in the control group ( $17.9 \%$ vs. $13.3 \%$, absolute difference 4.6 , $95 \%$ confidence interval 1.1 to $7.9 \%$ ). The probability of cognitive impairment accounting for the competing risk of death over 8 years was still not significantly different between the CVD and control groups ( $31.7 \%$ vs. $29.9 \%$, absolute difference 1.8 , 95\% confidence interval -2.4 to $6.2 \%$ ) (Supplementary Figure S3).

We performed an additional sensitivity analysis to examine the effects of altering the TICS-m score cutoff for cognitive impairment on the cumulative incidence of cognitive impairment in the CVD vs. control groups. Defining cognitive impairment as a TICS-m score of 12 or below, the probability of cognitive impairment over the 8-year study period increased to $38.9 \%$ in the CVD group (vs $29.7 \%$ in the original analysis) and $37.3 \%$ in the control group (versus $30.6 \%$ in the original analysis). Defining cognitive impairment as a TICS-m score of 10 or below, the probability of cognitive impairment over

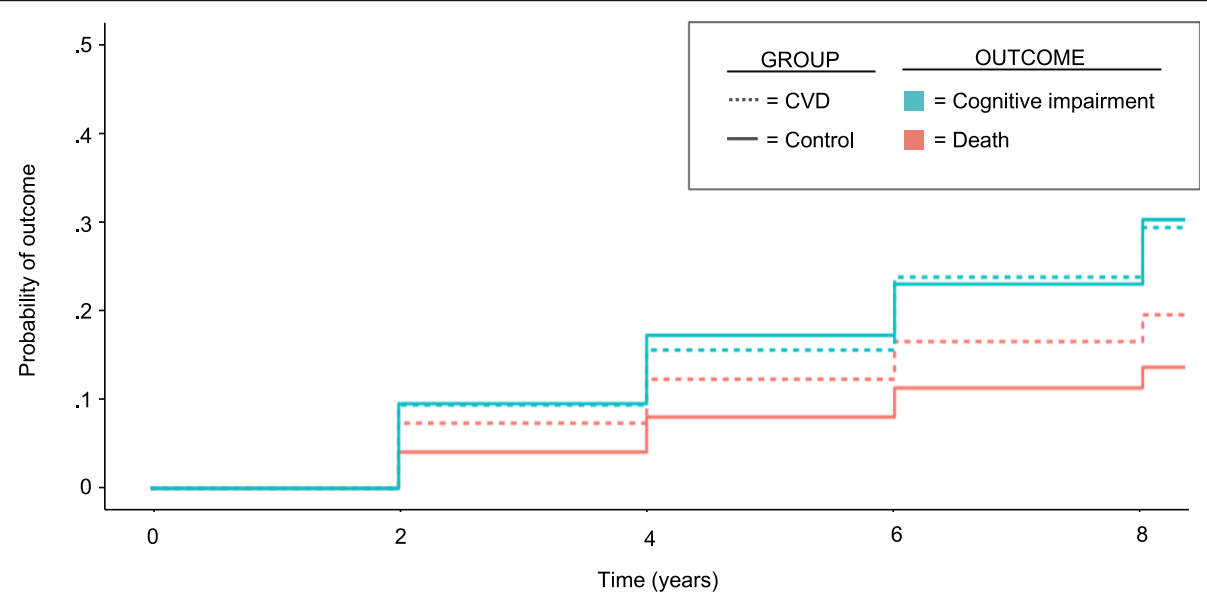

Fig. 2 Cumulative Incidence of Cognitive Impairment in CVD vs. Control Groups. Shown are the likelihood of death (orange) and the likelihood of cognitive impairment (blue) in the CVD group (dashed line) vs. control group (solid line) over the 8-year study period. Participants with CVD were more likely to experience death at follow-up than controls. There was no significant difference in the incidence of cognitive impairment between participants with CVD vs. controls 
Table 2 Effect of CVD and Covariates on Incidence of Cognitive Impairment per Subdistribution Hazard Regression Model

\begin{tabular}{ll}
\hline Variable & $\begin{array}{l}\text { Subdistribution Hazard Ratio } \\
\text { (95\% Confidence Interval) }\end{array}$ \\
\hline Incident CVD & $0.96(0.85-1.09)$ \\
Age (yrs) & $1.06(1.05-1.07)$ \\
Male gender & $1.10(0.97-1.25)$ \\
Race \& Ethnicity & \\
$\quad$ Non-Hispanic Black & $1.79(1.47-2.18)$ \\
Hispanic & $1.33(1.05-1.67)$ \\
Other & $1.45(0.93-2.28)$ \\
Education level & \\
$\quad$ Less than high school & $1.82(1.55-2.15)$ \\
High school & $1.38(1.21-1.58)$ \\
BMl (kg/m^2) & $0.99(0.97-1.00)$ \\
Married & $0.965(0.88-1.14)$ \\
Depressive symptoms & $1.23(1.03-1.48)$ \\
Smoking currently & $1.18(0.95-1.44)$ \\
Drinking behavior (past 3 mos) & \\
Not drinking & $0.909(0.52-1.62)$ \\
Low-risk drinker & $0.67(0.39-1.23)$ \\
Hypertension & $1.05(0.92-1.19)$ \\
Diabetes & $1.01(0.87-1.19)$ \\
Chronic lung disease & $0.94(0.76-1.15)$ \\
Cancer & $0.94(0.81-1.10)$ \\
\hline Race and &
\end{tabular}

Race and ethnicity is defined based on participants' primary response, and 'other' for race and ethnicity is defined as American Indian, Alaska Native, Asian, Native Hawaiian, Pacific Islander, or something else. Low-risk drinking is defined as 7 drinks or fewer per week or 3 drinks or fewer per day for women, or 14 drinks or fewer per week or 4 drinks or fewer per day for men on average over the past 3 months

Abbreviations: $C V D$ cardiovascular disease, $B M I$ body mass index

the 8-year study period decreased to $22.0 \%$ in the CVD group (versus $29.7 \%$ in the original analysis) and $20.5 \%$ in the control group (versus $30.6 \%$ in the original analysis). The probability of cognitive impairment accounting for the competing risk of death over 8 years was not significantly different between the CVD and control groups with a TICS-m cutoff of 12 (absolute difference 1.6, $95 \%$ confidence interval -3.1 to $6.0 \%$ ) or with a TICS-m cutoff of 10 (absolute difference 1.5, 95\% confidence interval -3.7 to $6.9 \%$ ) (Supplementary Table S2).

\section{Discussion}

In a large longitudinal study of older adults (mean age 73 years), we observed an increased probability of death but no increased probability of cognitive impairment among participants with incident CVD compared with an age- and gender-matched control group. This finding contradicts several recent studies indicating that incident
CVD accelerates cognitive decline [16-18]. However, there are several key differences between our study and previous studies that may account for our results. Firstly, our study utilized a cumulative incidence analysis accounting for the competing risk of death, which can be thought of as a prognostic model that examines the probability of cognitive impairment occurring in CVD vs. control patients in a real-world setting where death is a possibility [30]. This type of prognostic model that aims to predict likelihood is different than an etiological analysis that aims to infer the causal relationship between CVD and cognitive impairment. One logical interpretation of our results is that higher rates of death may preclude the development of cognitive impairment in a significant portion of the CVD population, as these participants may not live long enough after CVD diagnosis to develop significant cognitive decline. This is consistent with the idea that many of the proposed biologic mechanisms leading to cognitive impairment in CVD patients, such as systemic inflammation, oxidative damage, and subclinical vascular brain injury, are slow-acting processes that take many years to occur $[14,17]$.

Additionally, in measuring cognitive impairment, our study used a modified version of the TICS (TICS-m), which is a validated cognitive screening tool based on the Mini-Mental State Examination. While the TICS-m has high sensitivity and specificity for cognitive impairment in older adults [24, 25], it is designed to screen for cognitive impairment and does not provide the same degree of granularity as measuring specific domains of cognitive function, such as verbal memory, information processing speed, and temporal orientation, separately. Our results support the notion that CVD patients are not more likely than their non-CVD counterparts to experience global cognitive changes (that would clinically manifest as mild cognitive impairment or dementia) but do not speak to whether CVD patients have declines in cognitive performance in specific domains. Multiple previous studies examining accelerated cognitive decline in CVD patients have examined isolated cognitive domains rather than global cognitive status, and these studies have observed that CVD patients have faster cognitive decline in certain domains, such as verbal memory, information processing speed, and temporal orientation, but not in others, such as executive function and semantic fluency $[17,18]$. It is difficult to extrapolate whether these observed declines in specific cognitive domains would translate into global cognitive impairment that manifests clinically.

While the weight of prior evidence has suggested a connection between CVD and cognitive impairment, these findings are not universal. A meta-analysis conducted in 2017 found that taken together, prospective cohort studies showed increased risk of cognitive 
impairment in individuals with coronary heart disease, defined as individuals with angina pectoris and myocardial infarction, while cross-sectional and case-control studies did not [3]. Another review that analyzed longitudinal studies on cognitive impairment with atrial fibrillation, heart failure, peripheral artery disease, myocardial infarction, and impact of atherosclerotic burden separately found that atrial fibrillation and severe atherosclerosis were risk factors for cognitive decline but that the body of literature on heart failure, peripheral artery disease, and myocardial infarction was too small to draw any conclusions [8]. There is also significant heterogeneity across the literature in how CVD and cognitive impairment are defined and measured. Since CVD is a general term that can be comprised of many cardiovascular-related conditions (e.g. myocardial infarction, coronary artery disease, heart failure, arrythmia, stroke, etc.), studies have included different conditions in their analyses. Cognitive impairment is also a very broad term that can be defined and segmented in different ways (e.g. mild cognitive impairment vs. dementia, non-amnesiac cognitive impairment vs. amnesiac cognitive impairment), or examined by looking at domains of cognition (e.g. verbal memory, information processing speed, temporal orientation). This methodological heterogeneity makes it difficult to draw broader conclusions from the current body of literature. Further, it is also important to consider that bias towards publishing positive results may have played a role in shaping the current literature landscape.

Our study has several strengths, including a wellcharacterized dataset with a large sample size and serial cognitive assessments over nearly a decade of follow-up. Our study also has several important limitations. First, we relied on self-reported CVD. While we used an established HRS methodology to apply rigor to this process, we also found that participants with inconsistencies in self-report were more likely to have cognitive impairment, suggesting that cognitive status did play a role in participants' abilities to report on their cardiovascular disease status. However, our sensitivity analysis that included participants with self-report discrepancies still showed no difference in incidence of cognitive impairment between CVD and control groups. Utilizing self-report also resulted in a broader analysis of cognitive impairment in CVD overall, as opposed to a more focused analysis looking at cognitive impairment in one type of CVD, such as heart failure or myocardial infarction. While this limited the specificity of our analysis, prior research has proposed an association between cognitive impairment and the range of cardiac conditions, such as heart failure, myocardial infarction, atrial fibrillation, and coronary artery disease, that were included in our definition of CVD $[4,6,7,16,18]$.
A second limitation is that several variables were not available in in our dataset and may have influenced the results. For example, we lacked robust data on the severity of participants' CVD or how they managed their symptoms. It is possible that patients with more severe atherosclerotic disease burden were more likely to develop cognitive impairment. Third, we performed a clinical cutpoint-based analysis, rather than a continuous analysis of change in TICS-m, which limited our ability to detect differences between the CVD and control groups in the rate of decline in TICS-m or the association of incident CVD with TICS-m at the time of CVD onset. We decided on a cutpoint-based analysis to ease clinical interpretability, but we acknowledge the potential loss of information in doing so. Fourth, we used the Langa-Weir cognition dataset that included imputed values for participants missing some TICS responses. We employed this data to align with prior HRS studies and to minimize bias from exclusion of cognitively impaired participants, as not using these values leads to missing a large fraction of participants with dementia. However, we recognize that the imputation may have influenced results. Fifth, our sample included primarily non-Hispanic white participants, which does not reflect the diversity of race and ethnicity present in the U.S. population and limits the generalizability of our results. Sixth, we excluded a significant portion of the HRS population with CVD due to missing covariate data and age restrictions that were used to generate a sufficient matched cohort. Seventh, we did not control for presence of the ApoE4 allele, which some past studies on CVD and cognition accounted for given its strong association with dementia [34]. Lastly, we did not have data on specific cognitive domains, so we were unable to analyze cognitive impairment at a more granular level as other studies have done.

\section{Conclusions}

In summary, our results show an increased likelihood of death but not cognitive impairment among participants with incident CVD vs. an age- and gender-matched control group. From a clinical perspective, cognitive screening may still be useful in patients with CVD. There are many CVD patients with cognitive decline that impairs their ability to engage in self-care [35], and factors such as nonadherence to medication can lead to adverse outcomes. However, based on our findings and other negative studies [19-21], patients with CVD may not be a subgroup that requires targeted cognition screening beyond what is recommended for older adults more broadly.

\section{Abbreviations}

CVD: Cardiovascular disease; HRS: Health and retirement study; TICS$\mathrm{m}$ : Modified telephone interview for cognitive status 


\section{Supplementary Information}

The online version contains supplementary material available at https://doi. org/10.1186/s12877-021-02191-0.

Additional file 1: Supplementary Methods. Definition of CVD and Cognitive Impairment. Supplementary Table S1. Sample Size and Cohort Characteristics of CVD Group vs. Participants Excluded due to Selfreport Inconsistency. Supplementary Table S2. Change in Incidence of Cognitive Impairment with Varying TICS-m Score Cutoffs. Supplementary Figure S1. Flowchart of Participant Selection. Supplementary Figure S2. Distribution of Modified Telephone Interview for Cognitive Status Scores for CVD and Control Groups. Supplementary Figure S3. Cumulative Incidence of Cl in CVD Group Including Self-report Discrepancies vs. Control Group.

\section{Acknowledgements}

We would like to thank Dr. Simon Jones, PhD for providing guidance on generating matched samples in RStudio and Rosie Ferris, MPH for assistance with HRS data downloads and codebook interpretation.

\section{Authors' contributions}

Study design: A.C., J.D., L.H., and C.B. Data cleaning and analysis: A.C., S.S., and L.Y. Data interpretation: All authors. Preparation of manuscript: A.C. and J.D. Manuscript review: All authors. The authors read and approved the final manuscript.

\section{Funding}

This work was supported by the Medical Student Training in Aging Research program (5T35AG050998-05), which is sponsored by the National Institute on Aging to encourage medical students to consider a career in academic geriatrics, and a Patient Oriented Career Development Award (K23 AG052463) from the National Institute on Aging. The project used data from the Health and Retirement Study, which is supported by the National Institute on Aging (U01 AG009740) and the Social Security Administration.

\section{Availability of data and materials}

The datasets analyzed in this study are available in the Health and Retirement Study repository: https://hrs.isr.umich.edu/data-products?_ga=2. 9793781.1130511234.1607196676-1851121076.1607196676

\section{Declarations}

\section{Ethics approval and consent to participate}

This is a secondary analysis using the publicly available Health and Retirement Study. The Health and Retirement Study has been approved by the Institutional Review Board at the University of Michigan, adheres to methods that conform to all relevant guidelines and regulations, and obtains informed consent from voluntary participants. The public data, which is available to registered researchers, is de-identified.

\section{Consent for publication}

Not applicable.

\section{Competing interests}

No authors have declared competing interest for this article.

\section{Author details}

${ }^{1}$ New York University Grossman School of Medicine, 550 First Avenue, New York, NY, USA. ${ }^{2}$ Division of Healthcare Delivery Science, Department of Population Health, NYU Grossman School of Medicine, New York, NY, USA. ${ }^{3} \mathrm{NYU}$ Steinhardt School of Culture, Education, and Human Development, New York, NY, USA. ${ }^{4}$ National Committee for Quality Assurance, Washington, D.C., USA. ${ }^{5}$ Division of Biostatistics, Department of Population Health, NYU Grossman School of Medicine, New York, NY, USA. 'Leon H. Charney Division of Cardiology, Department of Medicine, NYU Grossman School of Medicine, New York, NY, USA.
Received: 6 December 2020 Accepted: 30 March 2021 Published online: 26 April 2021

\section{References}

1. Centers for Disease Control and Prevention. Heart Disease Facts. 2020 https://www.cdc.gov/heartdisease/facts.htm. Accessed 6 Dec 2020.

2. de Bruijn RF, Heeringa J, Wolters FJ, Franco OH, Stricker BH, Hofman A, et al. Association between atrial fibrillation and dementia in the general population. JAMA Neurol. 2015;72(11):1288-94. https://doi.org/10.1001/jama neurol.2015.2161.

3. Deckers K, Schievink SHJ, Rodriquez MMF, van Oostenbrugge RJ, van Boxtel MPJ, Verhey FRJ, et al. Coronary heart disease and risk for cognitive impairment or dementia: systematic review and meta-analysis. Plos One. 2017;12(9):e0184244. https://doi.org/10.1371/journal.pone.0184244.

4. Eggermont LH, de Boer K, Muller M, Jaschke AC, Kamp O, Scherder EJ. Cardiac disease and cognitive impairment: a systematic review. Heart. 2012; 98(18):1334-40. https://doi.org/10.1136/heartjnl-2012-301682.

5. Gure TR, Blaum CS, Giordani B, Koelling TM, Galecki A, Pressler SJ, et al. Prevalence of cognitive impairment in older adults with heart failure. J Am Geriatr Soc. 2012;60(9):1724-9. https://doi.org/10.1111/j.1532-5415.2012.04097.x.

6. Kalantarian S, Stern TA, Mansour M, Ruskin JN. Cognitive impairment associated with atrial fibrillation: a meta-analysis. Ann Intern Med. 2013;158(5 Pt 1):338-46. https://doi.org/10.7326/0003-4819-158-5-201303050-00007.

7. Lutski M, Weinstein G, Goldbourt U, Tanne D. Cardiovascular health and cognitive decline 2 decades later in men with preexisting coronary artery disease. Am J Cardiol. 2018;121(4):410-5. https://doi.org/10.1016/j.amjcard.2 017.11.003.

8. Stefanidis KB, Askew CD, Greaves K, Summers MJ. The effect of non-stroke cardiovascular disease states on risk for cognitive decline and dementia: a systematic and meta-analytic review. Neuropsychol Rev. 2018;28(1):1-15. https://doi.org/10.1007/s11065-017-9359-z.

9. Gruhn N, Larsen FS, Boesgaard S, Knudsen GM, Mortensen SA, Thomsen G, et al. Cerebral blood flow in patients with chronic heart failure before and after heart transplantation. Stroke. 2001;32(11):2530-3. https://doi.org/10.11 61/hs1101.098360.

10. Ikram MA, van Oijen M, de Jong FJ, Kors JA, Koudstaal PJ, Hofman A, et al. Unrecognized myocardial infarction in relation to risk of dementia and cerebral small vessel disease. Stroke. 2008;39(5):1421-6. https://doi.org/10.11 61/STROKEAHA.107.501106.

11. Pullicino PM, Hart J. Cognitive impairment in congestive heart failure?: embolism vs hypoperfusion. Neurology. 2001;57(11):1945-6. https://doi. org/10.1212/WNL.57.11.1945.

12. Redwine LS, Pung MA, Wilson K, Chinh K, Duffy AR. Differential peripheral inflammatory factors associated with cognitive function in patients with heart failure. Neuroimmunomodulation. 2018;25(3):146-52. https://doi.org/1 $0.1159 / 000493142$

13. Toledo C, Andrade DC, Diaz HS, Inestrosa NC, Del Rio R. Neurocognitive disorders in heart failure: novel pathophysiological mechanisms underpinning memory loss and learning impairment. Mol Neurobiol. 2019; 56(12):8035-51. https://doi.org/10.1007/s12035-019-01655-0.

14. Rivard L, Khairy P. Mechanisms, clinical significance, and prevention of cognitive impairment in patients with atrial fibrillation. Can J Cardiol. 2017; 33(12):1556-64. https://doi.org/10.1016/j.cjca.2017.09.024.

15. Schmidt R, Fazekas F, Offenbacher H, Dusleag J, Lechner H. Brain magnetic resonance imaging and neuropsychologic evaluation of patients with idiopathic dilated cardiomyopathy. Stroke. 1991;22(2):195-9. https://doi. org/10.1161/01.STR.22.2.195.

16. Hammond CA, Blades NJ, Chaudhry SI, Dodson JA, Longstreth WT Jr, Heckbert SR, et al. Long-term cognitive decline after newly diagnosed heart failure: longitudinal analysis in the CHS (cardiovascular health study). Circ Hear Fail. 2018;11(3):e004476. https:/doi.org/10.1161/circheartfailure.117.004476.

17. Schievink SHJ, van Boxtel MPJ, Deckers K, van Oostenbrugge RJ, Verhey FRJ, Kohler S. Cognitive changes in prevalent and incident cardiovascular disease: a 12-year follow-up in the Maastricht aging study (MAAS). Eur Heart J. 2017. https://doi.org/10.1093/eurheartj/ehx365.

18. Xie W, Zheng F, Yan L, Zhong B. Cognitive decline before and after incident coronary events. J Am Coll Cardiol. 2019;73(24):3041-50. https://doi.org/10.1 016/j.jacc.2019.04.019.

19. Bursi F, Rocca WA, Killian JM, Weston SA, Knopman DS, Jacobsen SJ, et al. Heart disease and dementia: a population-based study. Am J Epidemiol. 2006;163(2):135-41. https://doi.org/10.1093/aje/kwj025. 
20. Grubb NR, Simpson C, Fox KA. Memory function in patients with stable, moderate to severe cardiac failure. Am Heart J. 2000;140(1):E1-5. https:// www.sciencedirect.com/science/article/pii/S0002870300198333?via\%3Dihub. https://doi.org/10.1067/mhi.2000.106647.

21. Petrovitch H, White L, Masaki KH, Ross GW, Abbott RD, Rodriguez BL, et al. Influence of myocardial infarction, coronary artery bypass surgery, and stroke on cognitive impairment in late life. Am J Cardiol. 1998;81(8):1017-21. https://doi.org/10.1016/s0002-9149(98)00082-4.

22. Fisher $\mathrm{GG}$, Ryan $\mathrm{LH}$. Overview of the health and retirement study and introduction to the special issue. Work Aging Retire. 2018:4(1):1-9. https:// doi.org/10.1093/workar/wax032.

23. Cigolle CT, Nagel CL, Blaum CS, Liang J, Quinones AR. Inconsistency in the self-report of chronic diseases in panel surveys: developing an adjudication method for the health and retirement study. J Gerontol B Psychol Sci Soc Sci. 2018;73:901-12.

24. Plassman BL, Newman TT, Welsh KA, Helms M, et al. Properties of the telephone interview for cognitive status: Application in epidemiological and longitudinal studies. Neuropsychiatry Neuropsychol Behav Neurol. 1994;7:235-41.

25. de Jager CA, Budge MM, Clarke R. Utility of TICS-M for the assessment of cognitive function in older adults. Int J Geriatr Psychiatry. 2003;18(4):318-24. https://doi.org/10.1002/gps.830.

26. Crimmins EM, Kim JK, Langa KM, Weir DR. Assessment of cognition using surveys and neuropsychological assessment: the health and retirement study and the aging, demographics, and memory study. J Gerontol B Psychol Sci Soc Sci. 2011;66(Suppl 1):i162-71.

27. Langa KM, Weir DR, Kabeto M, Sonnega A. Langa-Weir classification of cognitive function (1995 onward); 2018.

28. Fisher GG, Hassan H, Faul JD, Rodgers WL, Weir DR. Health and Retirement Study Imputation of Cognitive Functioning Measures: 1992-2014; 2017.

29. Fine RJ, JPG. A proportional hazards model for the subdistribution of a competing risk. J Am Stat Assoc. 1999;94(446):496-509. https://doi.org/10.1 080/01621459.1999.10474144.

30. Noordzij M, Leffondre K, van Stralen KJ, Zoccali C, Dekker FW, Jager KJ. When do we need competing risks methods for survival analysis in nephrology? Nephrol Dial Transpl. 2013;28(11):2670-7. https://doi.org/10.1093/ndt/gft355.

31. RStudio Team. RStudio: integrated development for R. Boston: RStudio, PBC; 2020.

32. Gray RJ. Package 'cmprsk': subdistribution analysis of competing risks; 2019.

33. Austin PC. Practical recommendations for reporting Fine - Gray model analyses for competing risk data; 2017. p. 4391-400.

34. Liu C-C, Liu C-C, Kanekiyo T, Xu H, Bu G. Apolipoprotein E and Alzheimer disease: risk, mechanisms and therapy. Nat Rev Neurol. 2013;9(2):106-18. https://doi.org/10.1038/nrneurol.2012.263.

35. Dodson JA, Truong TT, Towle VR, Kerins G, Chaudhry SI. Cognitive impairment in older adults with heart failure: prevalence, documentation, and impact on outcomes. Am J Med. 2013;126(2):120-6. https://doi.org/10.1 016/j.amjmed.2012.05.029.

\section{Publisher's Note}

Springer Nature remains neutral with regard to jurisdictional claims in published maps and institutional affiliations.

Ready to submit your research? Choose BMC and benefit from:

- fast, convenient online submission

- thorough peer review by experienced researchers in your field

- rapid publication on acceptance

- support for research data, including large and complex data types

- gold Open Access which fosters wider collaboration and increased citations

- maximum visibility for your research: over $100 \mathrm{M}$ website views per year

At BMC, research is always in progress.

Learn more biomedcentral.com/submissions 